\title{
Sistema Brasileiro de Informação sobre Medicamentos - SISMED
}

\author{
The Brazilian Drug Information System - SISMED
}

Carlos C. F. Vidotti 1

Rogério Hoefler 1

Emília Vitória Silva 1

Gun Bergsten-Mendes 2

\footnotetext{
1 Centro Brasileiro

de Informação sobre Medicamentos, Conselho Federal de Farmácia. SBS Q.1, Bloco K, Ed. Seguradoras, 8 o andar, Brasília, DF 70093-900, Brasil. vidotti@tba.com.br 2 Departamento de Farmacologia, Faculdade de Ciências Médicas, Universidade Estadual de Campinas.

C.P. 6111, Campinas, SP 13081-970, Brasil. gbmendes@osite.com.br
}

\begin{abstract}
This paper describes the professional profile of pharmacists as specialists in drug information. Drug Information (DI) and Drug Information Centers (DIC) are defined. The fundamental activity of a DIC should be that of providing passive information or answering questions. The advantage of a DIC network is discussed, and strategies to implement the Brazilian Drug Information System (SISMED) are presented: investment in professional specialization and regular meetings of DIC coordinators to exchange experiences. The different DICs work within a cooperative protocol. Four training courses have been held, resulting in the rapid development of Brazil's national DIC network. Two national meetings of DIC professionals have helped strengthen the Brazilian Drug Information System.
\end{abstract}

Key words Drug Information; Drug Utilization; Drugs; Information Centers

Resumo Foram descritas as atividades do farmacêutico especialista em informação sobre medicamentos (IM) e caracterizados os Centros de Informação sobre Medicamentos (CIMs), deixando claro o que não são e o que são - mitos e fatos. Foram apresentadas as definições de IM e de CIMs e apresentadas as atividades usuais dos Centros, destacando a informação passiva como atividade fundamental. Foi apresentado o Sistema Brasileiro de Informação sobre Medicamentos (SISMED), uma rede de CIMs integrados através de um Protocolo de Cooperação que estabelece os requisitos mínimos para o funcionamento dos CIMs e os mecanismos de cooperação entre os Centros participantes. As estratégias para a formação do SISMED foram a capacitação de recursos humanos e encontros dos responsáveis pelos CIMs. O treinamento de recursos humanos através de quatro cursos, deu suporte à implantação rápida de CIMs pelo Brasil, preenchendo importante lacuna, e consolidando definitivamente esta atividade no país, dentro dos preceitos adotados mundialmente. Os encontros periódicos dos que trabalham nos CIMs fortaleceram os Centros, e consequentemente, o SISMED.

Palavras-chave Informação sobre Medicamentos; Uso de Medicamentos; Medicamentos; Centros de Informação 


\section{Introdução}

Paralelamente à introdução maciça de novos fármacos na terapêutica, aumentou exponencialmente a quantidade de informação sobre medicamentos, provocando o que se convencionou chamar de "explosão de informação", nem sempre de boa qualidade e imparcial. Entretanto este volume de dados não se difunde de modo eficiente e ágil, de modo que os profissionais da saúde têm dificuldade em se manter atualizados.

Uma solução proposta para este problema tem sido a formação de farmacêuticos clinicamente treinados como disseminadores de informação sobre medicamentos, serviço que estes profissionais, tanto comunitários como hospitalares, tradicionalmente vêm prestando de modo informal (Ascione et al., 1994). Nos Estados Unidos os farmacêuticos são os que mais atuam nos Centros de Informação sobre Medicamentos (CIMs) (Rosenberg et al., 1995), uma vez que, como categoria profissional, são eles que têm a formação mais abrangente sobre medicamentos (Ascione et al., 1994).

O termo "informação sobre medicamentos" foi desenvolvido no início dos anos 60, usado em conjunto com as palavras centro e especialista. Em 1962 foi criado o primeiro CIM, no Centro Médico da Universidade de Kentucky, nos Estados Unidos (Pellegrino, 1965; Amerson, 1996). A experiência teve sucesso e difundiu-se pelos Estados Unidos, depois pelo Canadá. Hoje existem centenas de Centros em todo o mundo (Miró \& Gutiérrez, 1992; Ascione et al., 1994; Bonati \& Tognoni, 1995).

A meta principal dos CIMs é a promoção do uso racional dos medicamentos. Para alcançar esta meta, a informação prestada deve ser objetiva, imparcial, imune a pressões políticas e econômicas. Ademais, como em muitos casos está em jogo a orientação para a situação clínica de um paciente, a informação deve ser fornecida com agilidade, em tempo hábil para sua utilização em cada caso.

O perfil profissional do especialista em informação sobre medicamentos, com treinamento e experiência clínica, deve incluir pelo menos as seguintes habilidades: competência na seleção, utilização e avaliação crítica da literatura; capacidade para apresentar a máxima informação relevante com um mínimo de documentação de suporte; conhecimento da disponibilidade de literatura, assim como de bibliotecas, centros de documentação, entre outros; capacidade de comunicar a informação farmacoterapêutica nas formas verbal e escrita; destreza no processamento eletrônico de dados; qualificação para participar nas Comissões de Farmácia e Terapêutica (OPS, 1995).

\section{Mitos}

O termo informação sobre medicamentos, vago e amplo, está geralmente voltado às diferentes questões ligadas à utilização dos medicamentos como indicação, posologia, compatibilidade, interações, reações adversas, estabilidade (Ascione et al., 1994; Malone et al., 1996; D’Aléssio et al., 1997).

Uma interpretação errônea consiste em comparar os serviços de um CIM com os de uma biblioteca, um centro de documentação, um banco de dados ou ainda um computador. Nunca, porém, estas fontes de informações incluem em seus serviços a contribuição de um especialista de informação sobre medicamentos e, muito menos, o fornecimento de informação dirigida à solução de problemas concretos ligados à utilização de medicamentos. Não são, portanto, alternativa para a informação que é obtida nos CIMs (OPS, 1995).

\section{Fatos}

Os CIMs, diferentemente das bibliotecas e dos centros de documentação, não proporcionam apenas documentos ou referências bibliográficas, mas soluções para problemas concretos sobre medicamentos ou para uma situação clínica de um paciente. Prestam informação selecionada, processada e avaliada por profissionais especializados, focalizada na necessidade particular do solicitante mediante um serviço altamente custo-efetivo (OPS, 1995; Kinky et al., 1999). Portanto, um CIM deve preencher, pelo menos, dois requisitos: contar com um farmacêutico especialista em informação sobre medicamentos, e ter bibliografia sobre medicamentos reconhecida internacionalmente, a mais atualizada possível.

\section{Definições}

Existe uma série de definições ligeiramente diferentes relativas à Informação sobre Medicamentos e Centro de Informação sobre Medicamentos, propostas por vários autores, em diferentes épocas e em diversos países (Molina \& Alberola, 1984; Ascione et al., 1994; OPS, 1995; Malone et al., 1996). A escolha de uma definição não invalida as demais. Foram escolhidas as de Malone et al. (1996:496) e de Molina \& 
Alberola (1984:6), respectivamente, expressas como:

- Informação sobre medicamentos (IM): "provisão de informação imparcial, bem referenciada e criticamente avaliada sobre qualquer aspecto da prática farmacêutica", e

- Centro de Informação sobre Medicamentos: "o lugar onde se realizam a seleção, a análise e a avaliação das fontes de informação sobre medicamentos, o que vai permitir a elaboração e a comunicação da informação desejada".

No II Encontro de Centros de Informação sobre Medicamentos do Brasil foi proposta uma outra definição: "CIM é o local que reúne, analisa, avalia e fornece informação sobre medicamentos, visando o seu uso racional" (Vidotti et al., 2000:28).

\section{Centro versus Serviço}

Os termos Centro de Informação sobre Medicamentos e Serviço de Informação sobre Medicamentos são às vezes usados como se fossem sinônimos embora existam diferenças. A literatura sugere que o primeiro deve denotar o local físico, e o último, a atividade desenvolvida neste local (Stanovich, 1996). Entretanto, para a finalidade estratégica de implantação da atividade de informação sobre medicamentos, é estabelecida uma hierarquia onde os Centros têm uma abrangência maior (por exemplo, um país, um estado, uma região), e os Serviços são institucionais, atendendo à demanda de um hospital (OPS, 1995; D’Alessio et al., 1997). Esta é a ótica adotada neste artigo, enfatizando-se, porém, que o importante é a atividade desenvolvida e não a sua designação, seja Centro ou Serviço.

O público alvo do CIM depende de vários fatores, entre os quais destacam-se: localização, capacidade de atendimento, infra-estrutura, horário de funcionamento, demanda, divulgação etc., com prioridade para os profissionais da saúde, podendo expandir-se para os usuários de medicamentos (Vidotti et al., 2000).

É importante salientar: o CIM deve ser divulgado de acordo com a capacidade de atendimento. Ou seja, o início de atividades deve ter um público-alvo crescente, mas a expansão deve ser gradativa, com auto-avaliações freqüentes. Primeiro é preciso desenvolver destreza para responder às questões que são formuladas ao CIM. O know-how e a credibilidade somente se adquirem com o tempo e a dedicação à atividade. Tudo deve ser feito dentro das possibilidades de cada Centro, pois, na medida em que o serviço requerido não for atendido com agilidade e qualidade, caminha-se a passos lar- gos para o descrédito do mesmo e da instituição que o comporta.

A Figura 1 relaciona as atividades de um Centro de Informação sobre Medicamentos, adaptado de Amerson (1996). Em relação a estas atividades é fundamental enfatizar dois aspectos:

- Não existe CIM sem a primeira atividaderesponder perguntas. Ou seja, ele pode desenvolver todas as demais atividades descritas, mas não será um CIM o lugar que desenvolver todas as atividades menos a primeira, e

- Responder perguntas deve representar a maior parte dos trabalhos prestados pelo CIM. Esta deve ser a atividade principal, devendo ser gratuita.

As atividades descritas na Figura 1 podem ser classificadas em duas grandes áreas (Molina \& Alberola, 1984):

- Informação passiva (reativa): é aquela oferecida em resposta à pergunta de um solicitante. $\mathrm{O}$ farmacêutico informador espera passivamente que o interessado lhe faça a pergunta. Desencadear a comunicação é iniciativa do solicitante.

- Informação ativa (proativa): é aquela em que a iniciativa da comunicação é do farmacêutico informador, o qual analisa que tipo de informação podem necessitar seus possíveis usuários (médicos, farmacêuticos, odontólogos, enfermeiros, pacientes, outros) e encontra uma via de comunicação para suprir estas necessidades.

\section{Sistema Brasileiro de Informação sobre Medicamentos (SISMED)}

A idéia de formação de uma rede de CIMs coordenada por um Centro de caráter nacional não é nova no contexto mundial (Calder et al., 1981; Skoutakis et al., 1987; Bonati \& Tognoni, 1995; Öhman, 1995). No Brasil, a primeira proposta foi feita em 1992 por um dos autores, exata-

Figura 1

Atividades de um Centro de Informação sobre Medicamentos.

Responder perguntas

Atividades da Comissão de Farmácia \& Terapêutica

Publicações: boletins, alertas, colunas em jornais

Educação: estágio, cursos sobre temas específicos da farmacoterapia

Revisão do uso de medicamentos

Atividades de pesquisa sobre medicamentos

Coordenação de programas de notificação (p. ex.: reações adversas a medicamentos)

Fonte: adaptado de Amerson, 1996. 
mente 30 anos depois que o primeiro CIM foi implantado no mundo, e está em desenvolvimento (Vidotti et al., 2000).

Embora seja uma atividade que começou a ser proposta há mais de 30 anos (Pellegrino, 1965), continua cada vez mais atual (Miró \& Gutiérrez, 1992; Bonati \& Tognoni, 1995; OPS, 1995). Conta hoje com as facilidades de um fantástico desenvolvimento tecnológico, incluídas aí as redes de informação, notadamente a Internet. Algumas das vantagens de uma rede de CIMs são: compartilhar recursos de informação; trocar experiências para a solução de problemas comuns e apoiar o esclarecimento de problemas ou casos difíceis; facilitar o desenvolvimento conjunto de produtos informativos; trocar e consolidar estatísticas identificando tendências em demanda de informação e promover o desenvolvimento de projetos de pesquisa em cooperação (OPS, 1995).

A rede brasileira de CIMs, de caráter não hierarquizado, descentralizada, formada por CIMs autônomos integrados por um Protocolo de Cooperação, é denominada de Sistema Brasileiro de Informação sobre Medicamentos (SISMED). Foi pensada tendo um Centro Nacional - o Centro Brasileiro de Informação sobre Medicamentos (CEBRIM), pertencente ao Conselho Federal de Farmácia (CFF), e Centros Regionais de Informação sobre Medicamentos (CRIM), um em cada estado. Sua criação foi resultado de esforço interinstitucional de caráter técnico-científico, com o propósito de apoiar a equipe multiprofissional da saúde, otimizando os recursos nesta área, e promovendo o uso racional de medicamentos. Poderá também apoiar o desenvolvimento da farmacoepidemiologia no país, com destaque para a farmacovigilância, que pode ser uma das atividades dos CIMs (Ascione et al., 1994; OPS, 1995; Amerson, 1996; D’Alessio et al., 1997).

\section{Estratégias para implantação e fortalecimento do SISMED}

As estratégias para formação e fortalecimento de uma rede de CIMs no Brasil previram a realização tanto de treinamento de farmacêuticos para implantação de CIM, como de reuniões periódicas entre os responsáveis pelos CIMs implantados.

\section{Treinamentos}

O CEBRIM, com apoio técnico e financeiro da Organização Pan-Americana da Saúde (OPAS), organizou quatro cursos com a finalidade de treinamento e capacitação de recursos humanos para a implantação, gestão e desenvolvimento de Centros de Informação sobre Medicamentos. Os cursos foram ministrados nos anos de 1994, 1995, 1996 e 1997, com a duração média de oito dias úteis, abertos às instituições de todo o país. Destacou-se neste treinamento a exigência de cada participante redigir um projeto para implantação de um CIM, adequado à sua realidade, que deveria ser apresentado formalmente à instituição de origem. Nesses quatro cursos foram treinados 53 farmacêuticos provenientes de 20 estados. Como resultado concreto desses treinamentos temos hoje 19 CIMs em 17 estados integrando o SISMED, através do Protocolo de Cooperação.

\section{Encontros}

As reuniões periódicas entre os CIMs, chamadas de Encontros, foram idealizadas para trocar experiências, uniformizar procedimentos, evidenciar estratégias de fortalecimento, buscar fontes de financiamento, identificar habilidades, realizar programas de pesquisa, executar atividades de educação continuada, produzir publicações, entre outras.

No ano de 1996 foi realizada a primeira reunião de CIMs, chamada de I Encontro de Centros de Informação sobre Medicamentos do Brasil, cujo objetivo era a consolidação do SISMED, através da aprovação do Protocolo de Cooperação, instrumento legitimado consensualmente pelos CIMs membros, que prevê a manutenção dos princípios básicos de um CIM; a normatização para os serviços dos CIMs; apoio técnico na criação e desenvolvimento de novos CIMs; evolução racional e integrada do Sistema.

Em 1998 foi realizado o II Encontro de Centros de Informação sobre Medicamentos do Brasil, quando foi indicado o Comitê Gestor do SISMED. Várias sugestões para o melhor desenvolvimento do SISMED foram propostas, entre as quais destacam-se: reconhecimento e apoio das instituições onde estão inseridos; maior apoio dos órgãos governamentais e da Organização Pan-Americana de Saúde (OPAS/ Brasil); busca de fontes de financiamento para os CIMs; harmonização das terminologias utilizadas na área (Vidotti et al., 2000).

\section{Perspectivas e desafios}

Os CIMs nasceram da necessidade de equacionar a grande quantidade de informação disponível sobre medicamentos e a aplicação destes 
conhecimentos à prática clínica. Atividades de ponta da profissão farmacêutica como a Farmácia Clínica, a Atenção Farmacêutica e a Farmacoepidemiologia têm nos CIMs um suporte técnico-científico inestimável. Fala-se hoje que o futuro da Farmácia, como profissão, passa por eles (Malone et al., 1996).

O SISMED foi proposto como forma de difundir a atividade no país e facilitar a implantação de novos centros, através de apoio técnico. Um grande desafio do SISMED é a implantação de CIMs em hospitais a fim de disponibilizar a informação sobre medicamentos no local onde ela é mais necessária. Outro desafio a ser enfrentado é a criação de CIMs especializados em áreas onde a informação é mais necessária como pediatria, geriatria, saúde da mulher, instalados principalmente em instituições hospitalares para atenderem à demanda local, mas também orientados à comunidade.

Qualquer que seja o lugar onde esteja instalado, o Centro deverá ser capaz de demonstrar o seu impacto social e econômico. O primeiro passo para isso é o desenvolvimento de programas de garantia de qualidade, com exemplos já descritos na literatura (Park \& Benderev, 1985). Este é um desafio a ser empreendido, do qual poderá depender a própria sobrevivência dos Centros.

A descrição deste passado recente deve ser útil para refletir sobre o futuro da atividade. $\mathrm{O}$ impacto social da promoção do uso racional de medicamentos torna a atividade necessária e atual, uma vez que em nada mudaram os fatores que levaram à sua implantação original. Além disso, a experiência do Brasil e de outros países demonstra o quanto o CIM é fundamental em uma sociedade que dispõe de cada vez mais medicamentos, muitos de uso complexo. No nosso país, além de tudo o mais, infelizmente, a desigualdade é muito grande em um mercado de medicamentos entre os dez maiores do mundo, pois a maior parte da população tem pequeno ou nenhum acesso a eles. Portanto, usá-los bem é um imperativo para todos e um desafio para os CIMs que, conceitualmente, se propõem a apoiar o uso racional dos medicamentos.

\section{Agradecimentos}

Ao Conselho Federal de Farmácia (CFF), na pessoa do Dr. Jaldo de Souza Santos, seu presidente. À Organização Pan-Americana da Saúde (OPAS), nas pessoas da Dra. Nelly Marin Jaramillo, Dr. Ileana R. Santich e Dr. Kees de Joncheere, assessores em Serviços Farmacêuticos da OPAS no Brasil, que têm nos apoiado desde 1992. A José Aleixo Prates e Silva e Thiers Ferreira, mentor e implementador desta atividade no CFF, respectivamente. A Vanessa B. de Paris e Edmundo Bond, do Centro de Información de Medicamentos (CEDIMED) (Caracas, Venezuela), Centro Colaborador da Organização Pan-Americana da Saúde/Organização Mundial de Saúde (OMS), nossos mestres.

\section{Referências}

AMERSON, A. B., 1996. Introduction to the concept of drug information. In: Drug Information. A Guide for Pharmacists (P. M. Malone, K. W. Mosdell, K. L. Kier \& J. E. Stanovich, eds.), pp. 3-11, Stamford: Appleton \& Lange.

ASCIONE, F. J.; MANIFOLD, C. C. \& PARENTI, M. A., 1994. Principles of Drug Information and Scientific Literature Evaluation. Hamilton: Drug Intelligence Publications.

BONATI, M. \& TOGNONI, G., 1995. Health Information Centres in Europe. Milan: Mario Negri Institute for Pharmacological Research. 
CALDER, G.; DAVIES, J. S.; McNUTTY, H. \& SMITH, J., 1981. Drug Information Network in the United Kingdom National Health Service. American Journal of Hospital Pharmacy, 38:663-666.

D’ALESSIO, R.; BUSTO, U. \& GIRON, N., 1997. Guía para el Desarrollo de Servicios Farmacéuticos Hospitalarios: Información de Medicamentos. Washington, D.C.: Organización Panamericana de la Salud.

KINKY, D. E.; ERUSH, S. C.; LASKIN, M. S. \& GIBSON, G. A., 1999. Economic impact of a drug information service. Annals of Pharmacotherapy, 33:11-16.

MALONE, P. M.; MOSDELL, K. W.; KIER, K. L. \& STANOVICH, J. E., 1996. Drug Information. A Guide for Pharmacists. Stamford: Appleton \& Lange.

MIRO, G. \& GUTIERREZ, L. M., 1992. Encuesta de centros de información de medicamentos en América Latina. Revista de la Organización de Farmacéuticos Ibero-Latinoamericanos, 2:301-308.

MOLINA, G. G. \& ALBEROLA, C., 1984. Información de medicamentos. Revista de la Asociación Española de Farmacéuticos Hospitalarios, 7:5-18.

OHMAN, B., 1995. Perspectives for a drug information centres network. In: Health Information Centres in Europe (M. Bonati \& G. Tognoni, eds.), pp. 38-41, Milan: Mario Negri Institute for Pharmacological Research.

OPS (Organización Panamericana de la Salud), 1995. Centros de Información de Medicamentos: Una Estrategia de Apoyo al Uso Racional de Medicamentos. Santo Domingo: Informe de Grupo de Trabajo Regional, OPS.
PARK, B. A. \& BENDEREV, K., 1985. Quality assurance program for a drug information center. American Journal of Hospital Pharmacy, 42:2180-2184.

PELLEGRINO, E. D., 1965. Drug information services and the clinician. American Journal of Hospital Pharmacy, 22:30-41.

ROSENBERG, J. M.; FUENTES, R. J.; STARR, C. H.; KIRSCHENBAUM, H. L. \& McGUIRE, H., 1995. Pharmacist-operated drug information centers in the United States. American Journal of HealthSystem Pharmacy, 52:991-996.

SKOUTAKIS, V. A.; WOJCIECHOWSKI, N. J.; CARTER, C. A.; HAYES, J. M.; HUDSON, B. L. \& MARTIN, J. A., 1987. Drug information center network: Need, effectiveness, and cost justification. Drug Intelligence and Clinical Pharmacy, 21:49-56.

STANOVICH, J. E., 1996. Drug information centers. In: Drug Information. A Guide for Pharmacists (P. M. Malone, K. W. Mosdell, K L. Kier \& J. E. Stanovich, eds.), pp. 345-355, Stamford: Appleton \& Lange.

VIDOTTI, C. C. F; HELEODORO, N. M.; ARRAIS, P. S. D.; HOEFLER, R.; MARTINS, R. \& CASTILHO, S. R., 2000. Centros de Informação sobre Medicamentos: Análise Diagnóstica no Brasil. Brasília: Conselho Federal de Farmácia/Organização PanAmericana de Saúde. 